\title{
Study of Electric and Mechanic Properties of the Implanted Artificial Cardiac Tissue Using a Whole Heart Model ${ }^{\star}$
}

\author{
Sándor Miklos Szilágyi ${ }^{1,2}$, László Szilágyi ${ }^{3}$, and Béat Hirsbrunner ${ }^{1}$ \\ 1 University of Fribourg, Fribourg, Switzerland \\ 2 Petru Maior University of Tîrgu-Mureş, Romania \\ szsandor72@yahoo.com \\ 3 Budapest University of Technology and Economics, Budapest, Hungary
}

\begin{abstract}
This study focuses on the effects of artificial cardiac tissue in the excitation-contraction process of the ventricular muscle. We developed a spatio-temporal computerized model of the whole heart that handles half millimeter sized compartments using 1 microsecond time step. We employed the effect of muscle fiber direction, laminar sheets, depolarization period and other parameters. The artificial tissue differs from the normal one in several ways, so their describing parameters are also modified. In our simulation the depolarization wave (DW) conduction speed of the artificial tissue was decreased by up to 3 times. In presence of a two centimeter wide and $2 \mathrm{~mm}$ thick artificial tissue slice, the maximal depolarization delay was $38 \mathrm{msec}$. Large ventricle size, low conducting speed and spaciousness of the injured ventricular tissue are the main generating factors of arrhythmia, while the location of the artificial tissue has secondary importance.
\end{abstract}

Keywords: ventricle modeling, geometry estimation, interpolation techniques.

\section{Introduction}

In developed countries, cardiac failure induced by myocardial infarction, despite several decades of research, still represents an important mortality factor. In spite of the advances in surgical techniques, immune system suppression and post-operative health-care, the life-saving and -extending effect of cardiac transplantation remains limited by shortage of proper donors and weakened immune system [1]. The development of proper artificial cardiac tissue (ACT) may eliminate both limiting factors [2].

As a first organ transplant substitution method, scientists have developed cell-based therapies, where special myocardial cells were injected into the tissue involved in infarction [3]. This approach was hardened by a massive apoptosis

* This work has been funded by the Scientific Exchange Program NMS-CH, "Rou: Swiss Research Fellowships", Project code 12031.

J. Ruiz-Shulcloper and G. Sanniti di Baja (Eds.): CIARP 2013, Part II, LNCS 8259, pp. 230-237, 2013.

(C) Springer-Verlag Berlin Heidelberg 2013 
of the injected cells (about 90\%) and the low rate of successfully differentiation into cardiomyocytes [4].

Nowadays the construction of a properly functioning "bio-artificial human heart" is still far away, but it became possible to create of three-dimensional (3D) muscle equivalents that can be useful for cardiac regeneration. As a result of continuous progress in the last two decades, the transplantation of contractile ACT and the replacement of degenerated tissue areas represent an important alternative to the whole organ transplantation [1]. Motivated to develop minimally invasive procedures, physicians' aim is to create biocompatible, non-immunogenic heart muscle that has similar morphological and functional properties as healthy cardiac tissue.

The myocardium, due to the cardiac progenitor cells [5], has a limited ability to recover after a serious injury, so the artificial tissue has to be surgically attached to the damaged area [6]. In order to introduce the new tissue in the ordinary work process, physicians have to create a proper capillary network [7]. The main obstacle of the integration of artificial tissue into the organ represents the cell apoptosis, caused by insufficient oxygen level in newly introduced tissue [8]. In the presence of this pathological condition, several parts of the artificial tissue may modify their electrical and mechanical properties that can develop altered depolarization and repolarization waves, causing rhythm irregularities [9]. The dysfunction of electrical impulse propagation may develop cardiac arrhythmias that perturb pump activity [10].

In the last decade several mathematical models and intelligent computational methods were developed in order to perform real-time computerized simulations of the whole heart, creating a useful tool to study cardiac dynamics [10 11]. These simulations have many advantages: they are not perturbed by data acquisition errors, the simulated values of all internal variables may be visualized, the size and nature of artificial tissue may be studied before the real intervention and the simulation may be stopped at any time for further improvements [12].

In the following we present the main benefits and dangers of the artificial tissue implantation procedure. Our goal is to establish a modeling platform that can a priori show the expected results of a future implantation. The negative effects of an eventually inaccurate operations can also a priori analysed.

The main goal of this paper is to model the onset of possible rhythm problems that can endanger the patient's life. The rest of the paper is organized as follows: Section 2 gives a detailed description of the cardiac excitation and contraction in presence of artificial tissue. Section 3 presents and discusses several aspects of modified depolarization and cardiac pump functionality, and the results of simulations. In Section 4 the conclusions are formulated.

\section{Materials and Methods}

\subsection{Modeling Background}

In our study we used a multi-level modeling technique that is visualized in Fig.

1. Each modeling level and the main descriptor parameters were described in 


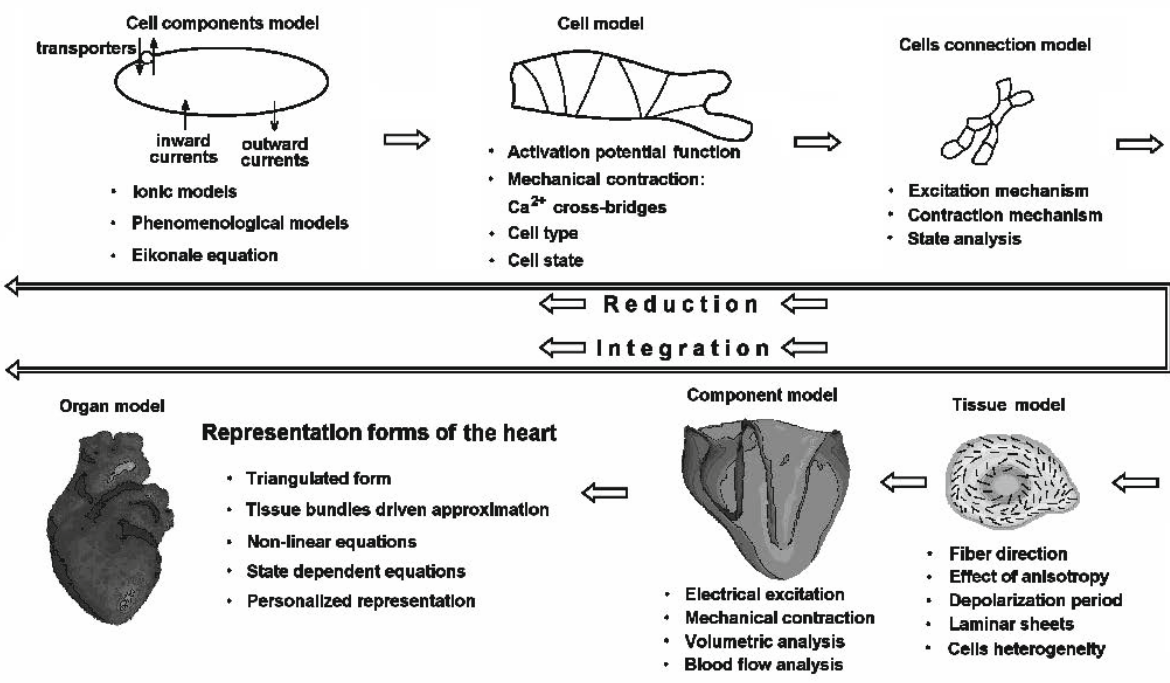

Fig. 1. The hierarchical structure of heart modeling, with all possible levels from individual cells to whole organ

our earlier work [12. As described in the above cited paper, the main modeling levels are: cell (type and state), cell connection, compartments, cardiac tissue (type, structure and state), component and whole organ.

In our simulation the lowest entity is considered a compartment that has a homogenous structure. These entities may contain only one cardiac cell type. Each compartment may be in normal or pathological state, which describes its electrical and mechanical behavior. All of these modeling units have unique activation potential function, mechanical contraction rules and a connection model that determines the propagation of the depolarization wave and the mechanical contraction of the cells. Moreover, the Purkinje system of the ventricles is also constituted by such compartments. These Purkinje units are also included in the connection system of the compartments.

As we move toward integration, we have to define the main properties of each integration element, such as: tissue, component and whole organ. The basic tissue parameters, such as fiber direction, anisotropy, depolarization period, laminar sheets and cell inhomogeneity are determined by its consisting compartments. The used component models enable us to determine the electrical excitation and mechanical contraction of the heart chambers, thus supporting the volumetric analysis and blood flow analysis for the given component or for the whole organ.

\subsection{The Main Properties of Artificial Cardiac Tissue}

The native cardiac tissue is a mixed structure of cardiac myocytes, fibroblasts, smooth muscle cells, endothelial cells and macrophages. The population of each 
cell type varies by state, age, gender and tissue's position. In our model the ratio and nature of excitable cells is defined in tissue properties, such as: fiber spatial orientation, level of anisotropy, shape of the activation potential that determines the depolarization period, laminar sheets, cells and structure inhomogeneity [12. Some biological properties are reflected indirectly in our model, for example the number and spreading manner of fibroblasts are not expressed directly but introduced in the level of conduction speed and level of anisotropy. These simplifications allow a much higher simulation speed, while the most important biological parameters are not altered notably.

In artificial tissues, the cellular complexity is drastically lower than in normal cardiac tissue. Several cell types are not present at all, and the overall structure is more homogeneous. The cellular complexity can be enhanced by using unpurified cardiac cell populations, but the cell arrangement cannot be controlled sufficiently. A partially controlled cellular arrangement not always yields better electro-mechanical properties instead of substantially higher cellular diversification.

The mechanical properties of the artificial tissues significantly differ from of the native ones. They usually are more sensitive to calcium regulation and may produce a lower absolute force than native cardiac tissue.

Nowadays all engineered cardiac tissues suffer from the absence of vascularization and perfusion. It is known that tumors cannot reach more than $3 \mathrm{~mm}$ diameter without capillarization. In case of cardiac muscle that makes permanent effort during contraction, the presence of sufficient nutrients and oxygen is imperial, so individual sheets cannot be thicker than $2 \mathrm{~mm}$. These layers can be partially vascularized after the implantation [13]. The construction of a thicker tissue layer demands the development of an inter-layer capillary system that nowadays is a challenging physiological task.

The connection area between the implanted artificial tissue slice and native myocardial cell allows significantly slower depolarization propagation than both native and implanted tissue.

\subsection{Details of the Simulation}

The compartment-based simulation uses an adaptive spatio-temporal resolution, so a $0.5 \mathrm{~mm}$ spatial and $1 \mu \mathrm{sec}$ temporal resolution is used in case of depolarization, and a significantly lower resolution (up to $5 \mathrm{~mm}$ and $1 \mathrm{msec}$ ) in resting phase. The instantaneous resolution, given for each region and time segment separately, depends on the derivative of the action potential (AP) function, connections of the simulated compartment, nature of studied phenomena and some restrictions implied by hardware or total simulation time considerations. The highest spatial and temporal resolution is needed at the depolarization wave's front line that propagates in an inhomogeneous and fast conducting tissue due to the fast voltage rise caused by fast sodium current [14]. The used spatio-temporal resolution may vary in time due other important factors, such as: simulation of various pathological cases especially arrhythmias, fragmentation of the depolarization front line and presence of spacious low- or non-conducting isles. 
The above presented compartment-based representation was used to simulate the electrical and mechanical behavior of all of cardiac cell types. The internal state of each compartment is modeled separately, which allows their investigation during the whole simulation. The connections among compartments are a priori determined, but its properties may vary with both space and time, so we can properly model the propagation of the depolarization wave and the mechanical contraction of the compartments in almost all circumstances.

Several time- and state-dependent tissue-related parameters were involved in our model that greatly influences the behavior of the compartment groups, such as: fiber direction, level of anisotropy, average depolarization period, laminar sheets and spontaneous cell inhomogeneity. The above mentioned parameters were deduced for each compartment separately from the simulation circumstances. The study of these parameters enables us to determine the electrical excitation and mechanical contraction of the cardiac muscle, thus supporting the volumetric analysis for atria and ventricles.

The tissue level excitation mechanism is based on Fast's work [15], but their results were transformed into compartment compatible data, considering each compartment as a secondary generator element, while the activation potential applied for ventricular tissue compartments was determined by using the LuoRudy II (LR) ventricular cell model [1617]. Each compartment may generate a depolarization wave if any adjacent elements are repolarized; otherwise, the propagation is swooned [12. The LR model accounts for dynamic changes of ionic concentrations, so it can properly handle several pathological cases. Although it contains few dozens of parameters instead of several hundreds used in newer ventricular models [11, the propagation of depolarization wave in the artificial tissue can be simulated properly.

During the simulation of a healthy cardiac activity, we employed the effect of: muscle fiber direction (the ratio between longitudinal and transversal conductivity varies from 2 to 10), normal and minimal depolarization period (considered 80-250 msec), laminar sheet effect (in-sheet transversal conduction 2-5 times faster than trans-sheet conduction), and cell inhomogeneity (using conduction speed differences for base-apex gradient (5\%-20\%), transmural epicardialendocardial gradient (5\%-35\%), left-right ventricular gradient (5\%-15\%)).

For pathological cases, normal parameter values were no longer maintained. In our simulation the depolarization wave (DW) conduction speed of the injuredbut still functioning-tissue was decreased by up to 20 times.

The simulation of various pathological circumstances, of the artificial tissue region or its barriers was performed using altered parameters [1819]. For example the effect of various anatomical modifications were considered as: muscle fiber direction (the ratio between longitudinal and transversal conductivity varies from 1 to 3 ), normal and minimal depolarization period (considered 70$350 \mathrm{msec}$ ), laminar sheet effect (in-sheet transversal conduction 1-2.5 times faster than trans-sheet conduction), and cell inhomogeneity (using conduction speed differences for base-apex gradient (0\%-25\%), transmural epicardial-endocardial gradient (0\%-50\%), left-right ventricular gradient (0\%-25\%)). 


\section{Results}

Fig. 2(left) presents the depolarization time of the ventricles in presence of a serious injury, covered by a two millimeter wide slice of artificial tissue. The injury is situated in the left paraseptal location. The depolarization time was calculated from the excitation moment of the atrio-ventricular (AV) node-HIS bundle system. The dark area visualizes the later excited tissue.
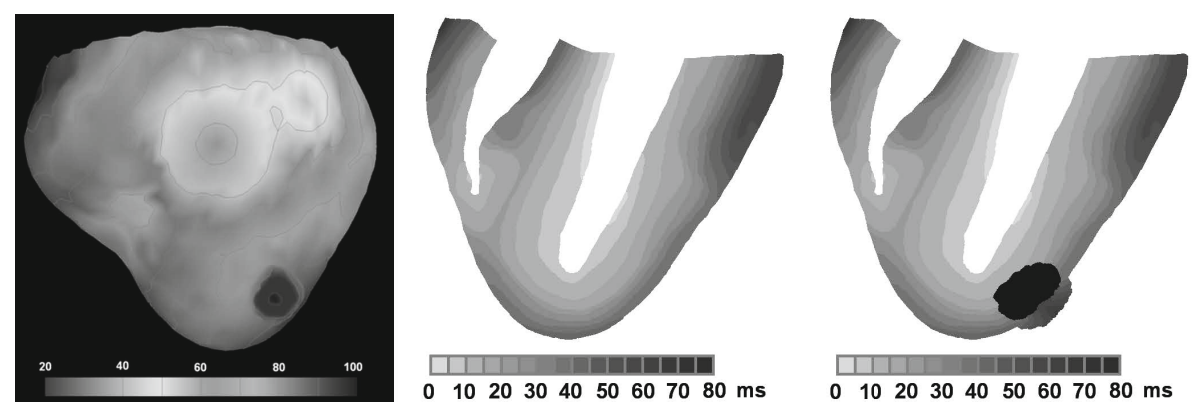

Fig. 2. (left) The depolarization time of the ventricles in presence of a $2 \mathrm{~mm}$ wide artificial tissue, implanted in top of the injured region situated in the left anterior paraseptal location. The depolarization time was determined from the excitation moment of the AVnode-HIS bundle system, and expressed in msec; (middle and right) The sectional representation of the depolarization time in ventricles. In the left side a normal ventricle is presented, while in the right side an anatomically similar ventricle had a serious injury, covered by a two millimeter wide slice of artificial tissue. The depolarization time was determined from the excitation moment of the AVnode - HIS bundle system. The sectional representation of the depolarization time in ventricles. In the left side a normal ventricle is presented, while in the right side an anatomically similar ventricle had a serious injury, covered by a two millimeter wide slice of artificial tissue. The depolarization time was determined from the excitation moment of the AVnode - HIS bundle system.

In the middle and right sections of Fig. 2, we get an insight into the depolarization phenomena of the inner ventricular structure, solved by a sectional representation. As shown in the figure, the depolarization time was counted from the excitation moment of the AV-node-HIS bundle system. In the middle section of Fig. 2] a healthy ventricular tissue is presented, while in the right section the ventricular tissue is seriously damaged, and is covered by a functioning slice of artificial tissue.

Table 1 presents the simulation results for healthy and injured tissue. The total depolarization time of the ventricle in presence of a $2 \mathrm{~cm}$ wide and 2 $\mathrm{mm}$ thick artificial tissue, situated in the right posterior region can reach 109 msec (counted from the excitation moment of the AV-node-HIS bundle system), while in case of a healthy ventricle the total depolarization time was $77 \mathrm{msec}$. The maximal depolarization delay highly depends on the size of the artificial tissue and slightly from the injured region. The $38 \mathrm{msec}$ maximal delay was 
Table 1. Simulated physiological parameters

\begin{tabular}{|c|c|c|c|}
\hline \multirow{2}{*}{$\begin{array}{c}\text { Case } \\
\text { study }\end{array}$} & Simulation parameters of the ventricles \\
\cline { 2 - 4 } & Studied phenomena & $\begin{array}{c}\text { Healthy } \\
\text { tissue }\end{array}$ & $\begin{array}{c}\text { Injury and } \\
\text { artificial tissue }\end{array}$ \\
\hline 1 & Total depolarization time & $77 \mathrm{msec}$ & $109 \mathrm{msec}$ \\
\hline 2 & Maximal depolarization delay & $0 \mathrm{msec}$ & $38 \mathrm{msec}$ \\
\hline 3 & Contractile efficiency & $100 \%$ & about $65 \%$ \\
\hline 4 & Maximal heartbeat rate (beats $/ \mathrm{min})$ & 300 & about 250 \\
\hline
\end{tabular}

determined for a $2 \mathrm{~cm}$ wide ACT situated in the posterior region, while the subjacent tissue was completely isolator. The contractile power of an injured ventricle with a $2 \mathrm{~cm}$ wide infarcted area in best case may reach half of the normal value. Our simulations show a $15 \%$ contractile power increase due the presence of ACT. Due to the presence of artificial tissue, de maximal heart bit rate was reduced by 50 beats per minute. It is important to mention that an altered shape of depolarization and repolarization may induce arrhythmias. As the heart rate becomes higher, the additional risk may drastically increase. The maximal heart beat was determined from the increase of the total cardiac depolarizationrepolarization cycle duration. We expected that ACT cells repolarize at least as fast as the middle ( $\mathrm{m}$ cells) of the ventricular tissue.

In case of high heart rate the delayed excitation of the ACT may induce irregular depolarization process that can develop various arrhythmias. The level of delayed excitation of ACT highly depends on its size and the nature of the subjacent tissue.

\section{Conclusions}

We created a simulation environment to show the effects of artificial tissue. The simulation was performed for a $2 \mathrm{~cm}$ wide and $2 \mathrm{~mm}$ thick artificial tissue. From the results of this simulation we concluded that the artificial tissue may enhance the cardiac pumping function, but also may increase the chance to develop arrhythmias. Numerical calculation confirms that occurring arrhythmia may develop ventricular fibrillation. This deadly phenomenon is promoted by diverse factors, such as: inhomogeneity in ventricular tissue, high excitation frequency, presence of accessory pathways, slow depolarization (due to thick walls) or repolarization and greater than normal ventricular size. Computerized simulation represents a non-invasive visualization tool than helps us understand the inner cardiac process in normal and pathological cases, and may help to select the most endangered patients that can enhance the efficiency of health care.

\section{References}

1. Kofidis, T., Akhyari, P., Boublik, J., Theodorou, P., Martin, U., Ruhparwar, A., et al.: In vitro engineering of heart muscle: Artificial myocardial tissue. J. Thorac. Cardiov. Sur. 124, 63-69 (2002) 
2. Venugopal, J.R., Prabhakaran, M.P., Mukherjee, S., Ravichandran, R., Dan, K., Ramakrishna, S.: Biomaterial strategies for alleviation of myocardial infarction. J. R. Soc. Interface 9(66), 1-19 (2012)

3. Heldman, A.W., Hare, J.M.: Cell therapy for myocardial infarction: Special delivery. J. Mol. Cell Cardiol. 44, 473-476 (2008)

4. Robey, T.E., Saiget, M.K., Reinecke, H., Murry, C.E.: Systems approaches to preventing transplanted cell death in cardiac repair. J. Mol. Cell Cardiol. 45, 567-581 (2008)

5. Jawad, H., Lyon, A.R., Harding, S.E., Ali, N.N., Boccaccini, A.R.: Myocardial tissue engineering. Brit. Med. Bull. 87, 31-47 (2008)

6. Miyagawa, S., Roth, M., Saito, A., Sawa, Y., Kostin, S.: Tissue-engineered cardiac constructs for cardiac repair. Ann. Thorac. Surg. 91, 320-329 (2011)

7. Radisic, M., Park, H., Gerecht, S., Cannizzaro, C., Langer, R., Vunjak-Novakovic, G.: Biomimetic approach to cardiac tissue engineering. Phil. Trans. R. Soc. B 362, 1357-1368 (2007)

8. Hool, L.C.: Acute hypoxia differentially regulates $\mathrm{K}(+)$ channels. Implications with respect to cardiac arrhythmia. Eur. Biophys. J. 34, 369-376 (2005)

9. Bueno-Orovio, A., Cherry, E.M., Fenton, F.H.: Minimal model for human ventricular action potentials in tissue. J. Theor. Biol. 253, 544-560 (2008)

10. Cherry, E.M., Fenton, F.H.: Visualization of spiral and scroll waves in simulated and experimental cardiac tissue. New J. Phys. 10, 125016 (2008)

11. ten Tusscher, K.H.W.J., Bernus, O., Hren, R., Panfilov, A.V.: Comparison of electrophysiological models for human ventricular cells and tissues. Prog. Biophys. Mol. Bio. 90, 326-345 (2006)

12. Szilágyi, S.M., Szilágyi, L., Benyó, Z.: A patient specific electro-mechanical model of the heart. Comput. Meth. Prog. Bio. 101, 183-200 (2011)

13. Leor, J., Aboulafia-Etzion, S., Dar, A., Shapiro, L., Barbash, I.M., Battler, A., Granot, Y., Cohen, S.: Bioengineered cardiac grafts: A new approach to repair the infarcted myocardium? Circulation 102(III), 56-61 (2000)

14. Cherry, E.M., Greenside, H.S., Henriquez, C.S.: A space-time adaptive method for simulating complex cardiac dynamics. Phys. Rev. Lett. 84, 1343-1346 (2000)

15. Fast, V.G., Rohr, S., Gillis, A.M., Kleber, A.G.: Activation of cardiac tissue by extracellular electrical shocks: formation of 'secondary sources' at intercellular clefts in monolayers of cultured myocytes. Circ. Res. 82, 375-385 (1998)

16. Luo, C.H., Rudy, Y.: A dynamic model of the cardiac ventricular action potential I. Simulations of ionic currents and concentration changes. Circ. Res. 74, 1071-1096 (1994)

17. Luo, C.H., Rudy, Y.: A dynamic model of the cardiac ventricular action potential. II. Afterdepolarizations, triggered activity, and potentiation. Circ. Res. 74, 1097-1113 (1994)

18. Rădoiu, D., Enăchescu, C., Adjei, O.: A systematic approach to scientific visualization. Eng. Comput. 23, 898-906 (2006)

19. Enăchescu, C.: Neural networks for function approximation. In: Int. Conf. BioInspired Comput. Meth. Used for Difficult Problem Solving (BICS 2008), pp. 84-89 (2008) 